\title{
Concentrically Layered Energy Equilibria of the Di-Block Copolymer Problem
}

\author{
Xiaofeng Ren * \\ Department of Mathematics and Statistics \\ Utah State University \\ Logan, UT 84322-3900, USA \\ Juncheng Wei ${ }^{\dagger}$ \\ Department of Mathematics \\ The Chinese University of Hong Kong \\ Shatin, Hong Kong
}

December, 2001

\begin{abstract}
We prove the existence of energy equilibria of the di-block copolymer problem in the unit disk. They consist of concentrically layered microdomains rich in one of the two monomer building units. We construct them by solving the proper singular limit of the free energy functional. The same limit also explains how under a dynamic law of the free energy, circular interfaces of non-equilibria may move to the origin and vanish, or collapse to each other, thereby reducing the number of layers.
\end{abstract}

\section{Introduction}

A di-block copolymer is a linear-chain molecule consisting of two sub-chains $a$ and $b$ grafted covalently to each other. The sub-chains $a$ and $b$ are made of different monomer units $A$ and $B$, respectively. In polymer systems even a weak repulsion between unlike monomers $A$ and $B$ induces a strong repulsion between $a$ and $b$. As a result the different sub-chains tend to segregate below some temperature $T_{c}$, but as they are chemically bonded, even a complete segregation of sub-chains $a$ and $b$ cannot lead to a macroscopic phase separation. Only a local micro-phase separation occurs: micro-domains rich in $A$ and $B$ are formed. See Bates and Fredrickson [2] for an introduction to block copolymers.

\footnotetext{
* Supported in part by NSF grant DMS-9703727.

† Supported in part by an Earmarked Grant of RGC, Hong Kong.
} 
A thermal equilibrium model based on the free energy was given by Leibler in [11] and Ohta and Kawasaki in [17]. Later Bahiana and Oono [1] derived this energy through a cell dynamical system approach. It takes the form

$$
F(u)=\int_{\Omega}\left[\frac{\epsilon^{2}}{2}|\nabla u|^{2}+W(u)+\frac{\sigma}{2}\left|(-\Delta)^{-1 / 2}(u-m)\right|^{2}\right] d x
$$

in the admissible set

$$
X_{m}=\left\{u \in W^{1,2}(\Omega): \frac{1}{|\Omega|} \int_{\Omega} u d x=m\right\},
$$

where $|\Omega|$ denotes the size of $\Omega$.

The two different monomer units are represented by $u=-1$ and $u=1$ respectively. The connectivity of the two monomer units leads to the long range interaction term $\frac{\sigma}{2}\left|(-\Delta)^{-1 / 2}(u-m)\right|^{2}$ in the free energy. Here $-\Delta$ is viewed as a positive operator, and $(-\Delta)^{-1 / 2}$ is the square root of its inverse. The parameter $\sigma$ is proportional to the inverse of the square root of the total chain length of the copolymer. $\frac{\epsilon^{2}}{2}|\nabla u|^{2}$ represents the interfacial energy density at bonding points. The parameter $\epsilon$ is proportional to the thickness of interfaces between the two monomers. $m$ stands for the average volume density of one of the two monomer units. It must be in the interval $(-1,1)$.

When this free energy is minimized, the three terms of the integrand (the energy density of the material) have different preferences. The first term likes large blocks of monomers, thereby reducing the combined size of interfaces between the two monomers. The function $W$ in the second term is a double well potential with two global minima at -1 and 1 , reflecting its preference for segregated monomers over mixtures. The third term is most interesting to us, which depends on $u$ nonlocally, through a global operator $(-\Delta)^{-1 / 2}$. Hence we call $F$ a nonlocal variational problem. The third term favours rapid oscillation between the two monomer. As these tendencies compete and compromise, small blocks rich with one of the two monomers appear. This phenomenon is known as micro-phase separation.

Equilibria of $F$ are critical points of (1.1), or solutions to its Euler-Lagrange equation:

$$
-\epsilon^{2} \Delta u+f(u)+\sigma(-\Delta)^{-1}(u-m)=\lambda \text { in } \Omega, \frac{\partial u}{\partial \nu}=0 \text { on } \partial \Omega .
$$

Here $f=W^{\prime}$, and $\lambda$ is a Lagrange multiplier. $(-\Delta)^{-1}(u-m)$ denotes the solution $v$ with the Neumann boundary condition and zero average of

$$
-\Delta v=u-m \text { in } \Omega, \partial_{\nu} v=0 \text { on } \partial \Omega, \int_{\Omega} v=0 .
$$

The precise meaning of $(-\Delta)^{-1 / 2}$ in $F$ is not too important for our purposes. It is the square root of the positive operator $(-\Delta)^{-1}$ from $\left\{w \in L^{2}(\Omega)\right.$ : $\left.\int_{\Omega} w d x=0\right\}$ to itself. Given $u \in X_{m}$, if we let $v=(-\Delta)^{-1}(u-m)$, then

$$
F(u)=\int_{\Omega}\left[\frac{\epsilon^{2}}{2}|\nabla u|^{2}+W(u)+\frac{\sigma}{2}|\nabla v|^{2}\right] d x
$$


which is often a more practical formula.

In this paper we continue to study $F$ following the work in $[19,20]$. The paper [19] was concerned with the one-dimensional version of the problem, $\Omega=(0,1)$, in the parameter range: $\sigma \sim \epsilon$. There we found a family of local minima of $F$. For each positive integer $N$, there are two local minima of $F$. Each of them assumes values close to -1 or 1 except on $N$ thin interfaces. In other words the copolymer forms $N+1$ micro-domains, separated by $N$ transition regions of mixtures. Here the micro-domains are of size 1 and the interfaces of size $\epsilon$. See Ohnishi et al [16] and Choksi [4] for more on the scales of micro-domains.

In [20] we studied the one-dimensional problem in the parameter range $\sigma \sim 1$. We proved that the global minima of $F$ are periodic functions whose periods have a particular asymptotic expansion in the order of $\epsilon^{1 / 3}$. These results extended the earlier ones of Müller [14] for the special case $m=0$ and $W(-u)=W(u)$. So in this parameter range the micro-domains are of size $\epsilon^{1 / 3}$, and the interfaces are of width $\epsilon$.

Of course the one-dimensional problem may be trivially extended to rectangles in $R^{2}$ or $R^{3}$, and the local and global minima obtained in one-dimension may be viewed as two or three-dimensional equilibria with parallel layers. A natural question is to study equilibria with curved interfaces.

In this paper we return to the parameter range $\epsilon \sim \sigma$ to address this issue. Taking $\Omega$ to be the unit disk $D$ in $R^{2}$, we look for equilibria of $F$ that are radially symmetric. Let us introduce

$$
\gamma=\frac{\sigma}{\epsilon}
$$

This $\gamma$ is a fixed constant, while both $\epsilon$ and $\sigma$ are small positive parameters.

Let $I_{\epsilon}$ be a scaled version of $F: I_{\epsilon}=\epsilon^{-1} F$, i.e.

$$
I_{\epsilon}(u)=\int_{D}\left[\frac{\epsilon}{2}|\nabla u|^{2}+\frac{1}{\epsilon} W(u)+\frac{\gamma}{2}\left|(-\Delta)^{-1 / 2}(u-m)\right|^{2}\right] d x .
$$

We remind the reader that $W$ is a smooth non-negative function assuming global minimum value 0 at \pm 1 . An example is $W(u)=\left(u^{2}-1\right)^{2}$. The derivative of $W$ is denoted by $f . m \in(-1,1)$ is a volume constraint on admissible functions $u$.

$I_{\epsilon}$ is defined in the radial function class

$$
X_{m}^{R}=\left\{u \in W^{1,2}(D): u(x)=u(|x|), \frac{1}{|D|} \int_{D} u d x=m\right\} .
$$

We seek equilibria of $I_{\epsilon}$ that are close to \pm 1 in $D$, except on one or more narrow circular interfaces. They represent copolymer configurations with concentrically layered monomer micro-domains.

Equilibria with a single circular interface are often called bubble solutions. When $\sigma=0, F_{\epsilon}$ becomes the more familiar Cahn-Hilliard problem [3]. The existence and location of bubble solutions were shown by Wei and Winter in [21]. However multiply layered radial equilibria of the type studied in this paper do not exist in the Cahn-Hilliard problem. 
We will construct both bubble solutions and multiply layered equilibria of $I_{\epsilon}$. The main results are contained in the following theorem.

Theorem 1.1 For any $\gamma>0$ defined in (1.2), there exist two equilibria of $I_{\epsilon}$ with one circular interface when $\epsilon$ is small. For $n \geq 2$ there exists $\gamma_{0}>0$ such that if $\gamma>\gamma_{0}$ there exist two equilibria of $I_{\epsilon}$ with $n$ circular interfaces when $\epsilon$ is small.

The largeness condition on $\gamma$ for the existence of several layer equilibria is new. In the one-dimensional case when $\epsilon$ is small there exist equilibria (actually local minima) with any prescribed number of layers for any $\gamma>0$ (See Theorem 1.1 of [19]). However in the higher dimensional case the curvature of layers interacts with the nonlocal effect in (1.3), and the condition on $\gamma$ becomes necessary.

Our strategy is to view $I_{\epsilon}$ for small $\epsilon$ as a singularly perturbed variational problem. After we find the singular limit: $I_{0}$, using the theory of $\Gamma$-convergence, we show that $I_{0}$ may be studied on a family of finite dimensional sets (See Proposition 2.3). In these sets we find strict local minima of $I_{0}$. They persist in the perturbed problem $I_{\epsilon}$.

We also study a dynamic law of the free energy $F$. We address the issue of interface disappearance. By studying a free boundary problem, we explain how interfaces may move to the origin and vanish, or collapse to each other.

Our paper is organized as follows: Section 2 identifies the singular limit $I_{0}$ of $I_{\epsilon}$; in Section 3 we solve $I_{0}$ and prove Theorem 1.1; Section 4 studies interface disappearance; a few remarks are included in Section 5.

\section{Reduction by $\Gamma$-convergence}

As illustrated in [19] when $\epsilon$ is small the study of $I_{\epsilon}$ can sometimes be reduced to its singular limit, known as the $\Gamma$-limit of $I_{\epsilon}$. It takes the form

$$
I_{0}(u)=c_{0}|D u|(D)+\frac{\gamma}{2} \int_{D}\left|(-\Delta)^{-1 / 2}(u-m)\right|^{2} d x
$$

where

$$
c_{0}=\frac{\sqrt{2}}{2} \int_{-1}^{1} \sqrt{W(s)} d s .
$$

$I_{0}$ is defined on the set

$$
X_{0, m}^{R}=\left\{u \in L^{2}(D): u(x)=u(|x|), \frac{1}{|D|} \int_{D} u d x=m\right\},
$$

although it has finite values only on a subset

$$
A=\left\{u \in X_{0, m}^{R}: u \in B V(D), u(x)= \pm 1 \text { a.e., }\right\} .
$$

$B V(D)$ is the space of functions on $D$ of bounded variation. On $X_{0, m}^{R} \backslash A$, $I_{0}=\infty$. For $u \in A I_{0}(u)$ is defined by $(2.1)$. 
In our two-dimensional case $|D u|(D)$ in (2.1) is twice the total length of the curves separating $\{x \in D: u(x)=-1\}$ from $\{x \in D: u(x)=-1\}$. More formally we view $|D u|$ as a finite measure and denote the size of $D$ under this measure by $|D u|(D)$. See Chapter 5 of [7] for more information on BV functions.

The general theory of $\Gamma$-convergence was developed by De Giorgi in [6]. A more complete introduction to this theory may be found in [5]. In the CahnHilliard problem, i.e. $\gamma=0$ in (1.3), this theory has been successfully applied by Modica and Mortola [13], Modica [12], Kohn and Sternberg [10]. The concept of $\Gamma$-convergence is defined by the two statements of the following proposition, which $I_{\epsilon}$ satisfies.

Proposition 2.1 1. For every family $\left\{u_{\epsilon}\right\} \subset X_{m}^{R}$ with $\lim _{\epsilon \rightarrow 0}\left\|u_{\epsilon}-u\right\|_{2}=0$,

$$
\liminf _{\epsilon \rightarrow 0} I_{\epsilon}\left(u_{\epsilon}\right) \geq I_{0}(u)
$$

2. For every $u \in A$, there exists a family $\left\{u_{\epsilon}\right\} \subset X_{m}^{R}$ such that $\lim _{\epsilon \rightarrow 0} \| u_{\epsilon}-$ $u \|_{2}=0$, and

$$
\limsup _{\epsilon \rightarrow 0} I_{\epsilon}\left(u_{\epsilon}\right) \leq I_{0}(u)
$$

The primary topology of $X_{0, m}^{R}$ is given by the $L^{2}$ norm, denoted by $\|\cdot\|_{2}$. Only one property of the $\Gamma$ convergence of $I_{\epsilon}$ to $I_{0}$ is needed for our purposes. It asserts that near every strict local minimum of $I_{0}$ there exists a local minimum of $I_{\epsilon}$ when $\epsilon$ is small. Denote the open ball in $X_{0, m}^{R}$, under the $L^{2}$-norm, centered at $u_{0}$ of radius $\delta$ by $B_{\delta}\left(u_{0}\right)$.

Proposition 2.2 Let $\delta>0$ and $u_{0} \in A$ be such that $I_{0}\left(u_{0}\right)<I_{0}(u)$ for all $u \in B_{\delta}\left(u_{0}\right)$ with $u \neq u_{0}$. Then there exists $\epsilon_{0}>0$ such that for all $\epsilon<\epsilon_{0}$ there exists $u_{\epsilon} \in B_{\delta / 2}\left(u_{0}\right)$ with $I_{\epsilon}\left(u_{\epsilon}\right) \leq I_{\epsilon}(u)$ for all $u \in B_{\delta / 2}\left(u_{0}\right)$. In addition $\lim _{\epsilon \rightarrow 0}\left\|u_{\epsilon}-u_{0}\right\|_{2}=0$.

The proofs of these two propositions are standard and may be found in Section 2 of [19].

Every $u \in A$ may be identified by the circular interfaces where $u$ jumps between -1 and 1 . Since the total measure $|D u|(D)$ is finite, there are finitely or countably infinitely many interfaces. If $u$ has infinitely many interfaces, they must converge to the origin. $A$ is naturally divided into two disjoint subsets $A^{-}$ and $A^{+}$. Every $u$ in $A^{-}$is equal to -1 outside the largest circular interface, and every $u$ in $A^{+}$is equal to 1 , outside the largest circular interface.

If we denote the radii of the circular interfaces of $u \in A^{ \pm}$by $r_{1}, r_{2}, \ldots, r_{k}, \ldots$ in the descending order, i.e. $1>r_{1}>r_{2}>\ldots>r_{k}>\ldots$, then $A^{ \pm}$may be identified by

$$
A^{ \pm}=\left\{\left(r_{1}, r_{2}, \ldots\right): 1>r_{1}>r_{2}>\ldots \geq 0, r_{1}^{2}-r_{2}^{2}+r_{3}^{2}-\ldots=\frac{1 \mp m}{2}\right\} .
$$

The constraint here follows directly from the constraint $\frac{1}{|\Omega|} \int_{\Omega} u d x=m$. 
Members in $A$ with finitely many interfaces are particularly important. Define

$$
A_{n}^{ \pm}=\left\{\left(r_{1}, r_{2}, \ldots\right) \in A^{ \pm}: r_{n+1}=r_{n+2}=\ldots=0\right\} .
$$

The $r_{i}$ 's are called the $r$-coordinates of $u$.

The subsets $A_{n}^{ \pm}$form an interesting structure in $A$. Both of $A_{1}^{ \pm}$contain a single element. Each of $A_{n}^{ \pm}, n \geq 2$, is open in the sense that it does not include its boundary. The boundary of $A_{2}^{-}$is the union of $A_{1}^{-}$and $A_{1}^{+}$. The boundary of $A_{2}^{+}$is also $A_{1}^{-} \cup A_{1}^{+}$. In general $A_{n}^{-}$and $A_{n}^{+}$share the same boundary: $\cup_{m<n}\left(A_{m}^{-} \cup A_{m}^{+}\right)$.

After factoring out a constant $\gamma \pi$, we decompose $I_{0}$ into a local part and a nonlocal part:

$$
I_{0}(u)=\gamma \pi(L(u)+N(u))
$$

with

$$
L(u)=c \sum_{k=1}^{\infty} r_{k}, N(u)=\int_{0}^{1}\left(v^{\prime}\right)^{2} r d r .
$$

Here $v=(-\Delta)^{-1}(u-m)$ and

$$
c=\frac{4 c_{0}}{\gamma}=\frac{2 \sqrt{2}}{\gamma} \int_{-1}^{1} \sqrt{W(s)} d s .
$$

When using the $r$-coordinate system, $I_{0}$ may be expressed as a function of $r_{1}, r_{2}, r_{3}, \ldots$ Let us do this for $u \in A^{-} . I_{0}$ in $A^{+}$may be calculated in a similar way and we leave the details to the reader.

Solving the equation for $v$, corresponding to $u$, we find that on each $\left(r_{i}, r_{i-1}\right)$

$$
v^{\prime}=\frac{c_{i}}{r}-\frac{( \pm 1-m) r}{2}
$$

The $c_{i}$ 's are determined by $v^{\prime}(0)=v^{\prime}(1)=0$ and the continuity of $v^{\prime}$ at $r_{i}$ :

$$
c_{1}=-\frac{1+m}{2}, c_{i}=r_{1}^{2}-r_{2}^{2}+\ldots+(-1)^{i} r_{i-1}^{2}-\frac{1+m}{2},(i=2,3, \ldots .)
$$

To see the expression for $I_{0}(u)$ in terms of the $r_{i}$ 's, we note that on $\left(r_{i}, r_{i-1}\right)$

$$
\begin{aligned}
\int_{r_{i}}^{r_{i-1}}\left(v^{\prime}\right)^{2} r d r= & c_{i}^{2} \log \frac{r_{i-1}}{r_{i}}-\frac{c_{i}\left((-1)^{i}-m\right)}{2}\left(r_{i-1}^{2}-r_{i}^{2}\right) \\
& +\frac{\left((-1)^{i}-m\right)^{2}}{16}\left(r_{i-1}^{4}-r_{i}^{4}\right),
\end{aligned}
$$

which leads to

$$
\begin{aligned}
I_{0}(u)= & \gamma \pi \sum_{i=1}^{\infty}\left\{c r_{i}+\left[\sum_{j=1}^{i-1}(-1)^{j-1} r_{j}^{2}-\frac{1+m}{2}\right]^{2} \log \frac{r_{i-1}}{r_{i}}\right. \\
& -\frac{\left[\sum_{j=1}^{i-1}(-1)^{j-1} r_{j}^{2}-\frac{1+m}{2}\right]\left((-1)^{i}-m\right)}{2}\left(r_{i-1}^{2}-r_{i}^{2}\right) \\
& \left.+\frac{\left((-1)^{i}-m\right)^{2}}{16}\left(r_{i-1}^{4}-r_{i}^{4}\right)\right\} .
\end{aligned}
$$


We call this expression $I_{0}(u)$ under the $r$-coordinate system. If $u \in A_{n}^{-}$, the sum becomes a finite one from 1 to $n+1$, with $r_{n+1}=0$.

It is often more convenient to use a different coordinate system for $u \in A^{-}$ (The case of $u \in A^{+}$may be handled similarly):

$$
y_{i}=\sum_{j=1}^{i}(-1)^{j-1} r_{j}^{2}=r_{1}^{2}-r_{2}^{2}+\ldots+(-1)^{i-1} r_{i}^{2},
$$

which we call the $y$-coordinates.

When $u$ has only finitely many interfaces, say $u \in A_{n}^{-}$,

$$
y_{n}=y_{n+1}=y_{n+2}=\ldots=\frac{1+m}{2} .
$$

So one advantage that the $y$-coordinate system enjoys over the $r$-coordinate system is that the $n-1$ degrees of freedom of $A_{n}^{-}$are all reflected in the first $n-1 y_{i}$ 's, without the need for a constraint as in (2.4).

The condition $r_{1}>r_{2}>r_{3} \ldots$ translates to

$$
1=y_{-1}>y_{1}>y_{3}>y_{5}>\ldots>\frac{1+m}{2}>\ldots>y_{6}>y_{4}>y_{2}>y_{0}=0
$$

for $y_{i}$ with $i \leq n-1$ if $u \in A_{n}^{-}$, or with $i$ an arbitrary positive integer if $u \in A^{-} \backslash A_{n}^{-}$. Note that

$$
c_{i}=y_{i-1}-\frac{1+m}{2}, r_{i}^{2}=(-1)^{i-1}\left(y_{i}-y_{i-1}\right) .
$$

Topologically the domain (2.9) of the $y_{i}$ 's is a diffeomorphism of $A_{n}^{-}$under the $r$-coordinates.

We may rewrite (2.8) under the $y$-coordinate system as

$$
\begin{aligned}
I_{0}(u)= & \gamma \pi \sum_{i=1}^{\infty}\left\{c \sqrt{\left|y_{i}-y_{i-1}\right|}+\frac{\left(y_{i-1}-\frac{1+m}{2}\right)^{2}}{2} \log \frac{y_{i-2}-y_{i-1}}{y_{i}-y_{i-1}}\right. \\
& +\frac{1-(-1)^{i} m}{2}\left(y_{i-1}-\frac{1+m}{2}\right)\left(y_{i-2}-y_{i}\right) \\
& \left.+\frac{\left[(-1)^{i}-m\right]^{2}}{16}\left(y_{i-2}^{2}-2 y_{i-2} y_{i-1}+2 y_{i-1} y_{i}-y_{i}^{2}\right)\right\} .
\end{aligned}
$$

We remind the reader that $y_{0}=0$ and $y_{-1}=-1$ by our convention. Again when $u \in A_{n}^{-}$, this sum becomes a finite one from 1 to $n+1$ with $y_{n}=y_{n+1}=$ $\frac{1+m}{2}$.

We close this section with an important proposition which allows us to seek local minima of $I_{0}$ in the finite dimensional sets $A_{n}^{ \pm}$.

Proposition 2.3 If $u$ is a strict local minimum of $I_{0}$ in $A_{n}^{-}$, or $A_{n}^{+}$for some $n \geq 1$, then $u$ is also a strict local minimum of $I_{0}$ in $X_{0, m}^{R}$. 
Proof. Suppose that $u$ is a strict local minimum of $I_{0}$ in $A_{n}^{-}$. Since $I_{0}=\infty$ outside $A$, it suffices to show that $u$ is a strict local minimum in $A$.

We do so by contradiction. Assume that there exists a sequence $u_{k} \in A$ such that $\left\|u_{k}-u\right\|_{2} \rightarrow 0$ and $I_{0}\left(u_{k}\right) \leq I_{0}(u)$.

Let us denote the radii of interfaces of $u_{k}$ by $r_{1}^{k}, r_{2}^{k}, r_{3}^{k}, \ldots$, and the radii of the interfaces of $u$ by $r_{1}, r_{2}, \ldots, r_{n}$. By the $L^{2}$ continuity of $N$ in $X_{0, m}^{R}$, $N\left(u_{k}\right) \rightarrow N(u)$. To accommodate both $I_{0}\left(u_{k}\right) \leq I_{0}(u)$ and $\left\|u_{k}-u\right\|_{2} \rightarrow 0$ we must have

$$
\lim _{k \rightarrow \infty} r_{j}^{k}=r_{j}, j=1,2, \ldots, n, \lim _{k \rightarrow \infty} \sum_{j=n+1}^{\infty} r_{j}^{k}=0, j=n+1, n+2, \ldots
$$

The condition $\left\|u_{k}-u\right\|_{2} \rightarrow 0$ also implies

$$
\lim _{k \rightarrow \infty} \sum_{j=1}^{\infty}\left[\left(r_{n+2 j-1}^{k}\right)^{2}-\left(r_{n+2 j}^{k}\right)^{2}\right]=0
$$

They imply that in the $y$-coordinates

$$
\lim _{k \rightarrow \infty} y_{j}^{k}=y_{j}, j=1,2, \ldots, n-1, \lim _{k \rightarrow \infty} y_{j}^{k}=\frac{1+m}{2}, j=n, n+2, \ldots
$$

uniformly in $j$. Here $y_{j}^{k}, j=1,2,3 \ldots$, are the $y$-coordinates of $u_{k}$, and $y_{j}$, $j=1,2, \ldots, n-1$, the $y$-coordinates of $u$.

Next we define $w_{k} \in A_{n}^{-}$, with $y$-coordinates $\left(y_{1}^{k}, y_{2}^{k}, \ldots, y_{n-1}^{k}, \frac{1+m}{2}, \frac{1+m}{2}, \ldots\right)$. Of the $n$ interfaces that $w_{k}$ has the first $n-1$ are shared with those of $u_{k}$. The $n$-th one of $w_{k}$ is slightly different from that of $u_{k}$. Clearly $\left\|w_{k}-u\right\|_{2} \rightarrow 0$. We show that $I_{0}\left(u_{k}\right) \geq I_{0}\left(w_{k}\right)$. Break $\frac{1}{\gamma \pi}\left(I_{0}\left(u_{k}\right)-I_{0}\left(w_{k}\right)\right)$ into three parts: $T_{1}, T_{2}$, and $T_{3}$, where

$$
\begin{aligned}
T_{1}= & \sum_{i=n+2}^{\infty}\left\{c \sqrt{\left|y_{i}^{k}-y_{i-1}^{k}\right|}+\frac{\left(y_{i-1}^{k}-\frac{1+m}{2}\right)^{2}}{2} \log \frac{y_{i-2}^{k}-y_{i-1}^{k}}{y_{i}^{k}-y_{i-1}^{k}}\right. \\
& +\frac{1-(-1)^{i} m}{2}\left(y_{i-1}^{k}-\frac{1+m}{2}\right)\left(y_{i-2}^{k}-y_{i}^{k}\right) \\
& \left.+\frac{\left((-1)^{i}-m\right)^{2}}{16}\left(\left(y_{i-2}^{k}\right)^{2}-2 y_{i-2}^{k} y_{i-1}^{k}+2 y_{i-1}^{k} y_{i}^{k}-\left(y_{i}^{k}\right)^{2}\right)\right\}, \\
T_{2}= & \left\{c \sqrt{\left|y_{n+1}^{k}-y_{n}^{k}\right|}+\frac{\left(y_{n}^{k}-\frac{1+m}{2}\right)^{2}}{2} \log \frac{y_{n-1}^{k}-y_{n}^{k}}{y_{n+1}^{k}-y_{n}^{k}}\right. \\
& +\frac{1-(-1)^{n+1} m}{2}\left(y_{n}^{k}-\frac{1+m}{2}\right)\left(y_{n-1}^{k}-y_{n+1}^{k}\right) \\
& \left.+\frac{\left((-1)^{n+1}-m\right)^{2}}{16}\left(\left(y_{n-1}^{k}\right)^{2}-2 y_{n-1}^{k} y_{n}^{k}+2 y_{n}^{k} y_{n+1}^{k}-\left(y_{n+1}^{k}\right)^{2}\right)\right\} \\
& -\frac{\left((-1)^{n+1}-m\right)^{2}}{16}\left(y_{n-1}^{k}-\frac{1+m}{2}\right)^{2}, \\
T_{3}= & \left\{c \sqrt{\left|y_{n}^{k}-y_{n-1}^{k}\right|}+\frac{\left(y_{n-1}^{k}-\frac{1+m}{2}\right)^{2}}{2} \log \frac{y_{n-2}^{k}-y_{n-1}^{k}}{y_{n}^{k}-y_{n-1}^{k}}\right.
\end{aligned}
$$




$$
\begin{aligned}
& +\frac{1-(-1)^{n} m}{2}\left(y_{n-1}^{k}-\frac{1+m}{2}\right)\left(y_{n-2}^{k}-y_{n}^{k}\right) \\
& \left.+\frac{\left((-1)^{n}-m\right)^{2}}{16}\left(\left(y_{n-2}^{k}\right)^{2}-2 y_{n-2}^{k} y_{n-1}^{k}+2 y_{n-1}^{k} y_{n}^{k}-\left(y_{n}^{k}\right)^{2}\right)\right\} \\
& -\left\{c \sqrt{\left|\frac{1+m}{2}-y_{n-1}^{k}\right|}+\frac{\left(y_{n-1}^{k}-\frac{1+m}{2}\right)^{2}}{2} \log \frac{y_{n-2}^{k}-y_{n-1}^{k}}{\frac{1+m}{2}-y_{n-1}^{k}}\right. \\
& +\frac{1-(-1)^{n} m}{2}\left(y_{n-1}^{k}-\frac{1+m}{2}\right)\left(y_{n-2}^{k}-\frac{1+m}{2}\right) \\
& \left.+\frac{\left((-1)^{n}-m\right)^{2}}{16}\left(\left(y_{n-2}^{k}\right)^{2}-2 y_{n-2}^{k} y_{n-1}^{k}+2 y_{n-1}^{k}\left(\frac{1+m}{2}\right)-\left(\frac{1+m}{2}\right)^{2}\right)\right\} .
\end{aligned}
$$

According to the computation leading to (2.8-2.10) each term in the sum of $T_{1}$ is simply

$$
c r_{i}^{k}+\int_{r_{i}^{k}}^{r_{i-1}^{k}}\left(v_{k}^{\prime}\right)^{2} r d r \geq 0
$$

where $v_{k}^{\prime}=(-\Delta)^{-1}\left(u_{k}-m\right)$. Hence $T_{1} \geq 0$.

We rewrite $T_{2}$ as

$$
\begin{aligned}
T_{2}= & c \sqrt{\left|y_{n+1}^{k}-y_{n}^{k}\right|}+\frac{\left(y_{n}^{k}-\frac{1+m}{2}\right)^{2}}{2} \log \frac{y_{n-1}^{k}-y_{n}^{k}}{y_{n+1}^{k}-y_{n}^{k}} \\
& +\frac{1-(-1)^{n+1} m}{2}\left(y_{n}^{k}-\frac{1+m}{2}\right)\left(y_{n-1}^{k}-y_{n+1}^{k}\right)+\frac{\left((-1)^{n+1}-m\right)^{2}}{2} \\
& \cdot\left[-2 y_{n-1}^{k}\left(y_{n}^{k}-\frac{1+m}{2}\right)+2 y_{n}^{k} y_{n+1}^{k}-\left(y_{n+1}^{k}\right)^{2}-\left(\frac{1+m}{2}\right)^{2}\right] .
\end{aligned}
$$

Since $\left|y_{n}^{k}-\frac{1+m}{2}\right|<\left|y_{n+1}^{k}-y_{n}^{k}\right|$, we may bound the last three terms below by $-C_{1}\left|\left(y_{n+1}^{k}-y_{n}^{k}\right) \log \left(y_{n+1}^{k}-y_{n}^{k}\right)\right|$, which is in turn controlled by the first term. For the sake of the next estimate, we save half of the first term and deduce that for large $k$

$$
\begin{aligned}
T_{2} & \geq c \sqrt{\left|y_{n+1}^{k}-y_{n}^{k}\right|}-C_{1}\left|\left(y_{n+1}^{k}-y_{n}^{k}\right) \log \left(y_{n+1}^{k}-y_{n}^{k}\right)\right| \\
& \geq \frac{c}{2} \sqrt{\left|y_{n+1}^{k}-y_{n}^{k}\right|} .
\end{aligned}
$$

For $T_{3}$ we note that

$$
\left(y_{n-1}^{k}-\frac{1+m}{2}\right)\left(y_{n-2}^{k}-y_{n}^{k}\right)-\left(y_{n-1}^{k}-\frac{1+m}{2}\right)\left(y_{n-2}^{k}-\frac{1+m}{2}\right) \geq 0
$$

by (2.9). This yields

$$
\begin{aligned}
T_{3} \geq & c\left(\sqrt{\left|y_{n}^{k}-y_{n-1}^{k}\right|}-\sqrt{\left|\frac{1+m}{2}-y_{n-1}^{k}\right|}\right) \\
& +\frac{\left(y_{n-1}^{k}-\frac{1+m}{2}\right)^{2}}{2}\left[\log \left|\frac{1+m}{2}-y_{n-1}^{k}\right|-\log \left|y_{n}^{k}-y_{n-1}^{k}\right|\right] \\
& +\frac{\left((-1)^{n}-m\right)^{2}}{16}\left[2 y_{n-1}^{k}-y_{n}^{k}-\frac{1+m}{2}\right]\left(y_{n}^{k}-\frac{1+m}{2}\right) .
\end{aligned}
$$


Since both $y_{n-1}^{k}-y_{n}^{k}$ and $y_{n-1}^{k}-\frac{1+m}{2} \rightarrow y_{n-1}-\frac{1+m}{2} \neq 0$, the mean value theorem is applied to the square root and logarithmic functions to conclude that $T_{3}$ is bounded below by $-C_{2}\left|y_{n}^{k}-\frac{1+m}{2}\right|$. Again by (2.9) $\left|y_{n}^{k}-\frac{1+m}{2}\right|<\left|y_{n}^{k}-y_{n+1}^{k}\right|$, so we use what we have saved in (2.12) to conclude that as $\left|y_{n+1}^{k}-y_{n}^{k}\right| \rightarrow 0$,

$$
T_{2}+T_{3} \geq \frac{c}{2} \sqrt{\left|y_{n+1}^{k}-y_{n}^{k}\right|}-C_{2}\left|y_{n+1}^{k}-y_{n}^{k}\right| \geq 0 .
$$

Once having proved $I_{0}\left(u_{k}\right) \geq I_{0}\left(w_{k}\right)$, we recall that $u$ is a strict local minimum in $A_{n}^{-}$and $w_{k} \in A_{n}^{-}$approaches $u$. So $I_{0}\left(u_{k}\right)>I_{0}(u)$, contradicting the assumption at the start of the proof.

\section{Local minima of $I_{0}$ in $A_{n}^{ \pm}$}

Proposition 2.3 suggests that we seek local minima of $I_{0}$ in $A_{n}^{ \pm}$. We do so in this section. Recall that for elements in $A_{n}^{ \pm}$, only their $y_{1}, y_{2}, \ldots, y_{n-1}$ coordinates are variables, and $y_{n}=y_{n+1}=\ldots=\frac{1+m}{2}$ are constants. It is convenient to introduce

$$
p_{i}=1-(-1)^{i} m \text {. }
$$

The nonlocal part $N$ of $I_{0}$, defined in (2.6), is the focus of this section.

Proposition 3.1 In each $A_{n}^{ \pm} N$ has a unique critical point $\left(y_{1}, y_{2}, \ldots, y_{n-1}\right)$ which satisfies

$$
p_{0} y_{n}>p_{1}\left(y_{1}-y_{n}\right)>p_{2}\left(y_{n}-y_{2}\right)>\ldots>(-1)^{n-1} p_{n-1}\left(y_{n}-y_{n-1}\right)>0 .
$$

Proof. In each of $A_{1}^{ \pm}$there is only one element, which is considered trivially a critical point.

In $A_{n}^{-}(n \geq 2)$ the derivatives of $N$ with respect to the $y_{i}$ 's are

$$
\begin{aligned}
\frac{\partial N}{\partial y_{1}} & =\frac{p_{2}\left(-y_{2}\right)}{2}+\left(y_{1}-y_{n}\right) \log \frac{-y_{1}}{y_{2}-y_{1}}, \\
\frac{\partial N}{\partial y_{i}} & =\frac{p_{i+1}\left(y_{i-1}-y_{i+1}\right)}{2}+\left(y_{i}-y_{n}\right) \log \frac{y_{i-1}-y_{i}}{y_{i+1}-y_{i}}, \\
\frac{\partial N}{\partial y_{n-1}} & =\frac{p_{n}\left(y_{n-2}-y_{n}\right)}{2}+\left(y_{n-1}-y_{n}\right) \log \frac{y_{n-2}-y_{n-1}}{y_{n}-y_{n-1}} .
\end{aligned}
$$

If $\left(y_{1}, y_{2}, \ldots, y_{n-1}\right)$ is a critical point, set

$$
z_{n-1}=\frac{y_{n-2}-y_{n}}{y_{n}-y_{n-1}}>0 .
$$

Then $\frac{\partial N}{\partial y_{n-1}}=0$ implies

$$
\frac{p_{n}}{2} z_{n-1}=\log \left(1+z_{n-1}\right)
$$


which is solvable for $z_{n-1}>0$ if and only if $\frac{p_{n}}{2}<1$, and it is true since $m \in(-1,1)$. So there exists a unique $z_{n-1}$ solely determined by (3.3). It depends on $m$ only.

Next we let

$$
z_{n-2}=\frac{y_{n-3}-y_{n-1}}{y_{n-1}-y_{n-2}}>0 .
$$

The condition $\frac{\partial N}{\partial y_{n-2}}=0$ implies

$$
\frac{p_{n-1}}{2} \frac{y_{n-1}-y_{n-2}}{y_{n}-y_{n-2}} z_{n-2}=\log \left(1+z_{n-2}\right) .
$$

By (3.2) it is $z_{n-1}$ rather than $y_{n-1}$ and $y_{n-2}$ that determines this equation. To have a positive solution of $z_{n-2}$ we must have $\frac{p_{n-1}}{2} \frac{y_{n-1}-y_{n-2}}{y_{n}-y_{n-2}}<1$, or equivalently

$$
\frac{p_{n-1}\left(y_{n-1}-y_{n}\right)}{p_{n-2}\left(y_{n}-y_{n-2}\right)}<1
$$

Because of $(3.2),(3.6)$ is indeed a requirement solely on $z_{n-1}: z_{n-1}>\frac{p_{n-1}}{p_{n-2}}$. To verify this condition, we recall the equation (3.3) that determines $z_{n-1}$. We must have

or equivalently

$$
\frac{p_{n}}{2} \frac{p_{n-1}}{p_{n-2}}<\log \left(1+\frac{p_{n-1}}{p_{n-2}}\right),
$$

$$
\log \left(1-\frac{p_{n-1}}{2}\right)<-\frac{p_{n-1}}{2}
$$

which is true since $p_{n-1} \neq 0$. Thus we have obtained a unique $z_{n-2}$, determined from (3.5), depending on $m$ and $z_{n-1}$ only.

In order to facilitate induction, we show that

$$
\frac{p_{n-2}\left(y_{n-2}-y_{n}\right)}{p_{n-3}\left(y_{n}-y_{n-3}\right)}<1
$$

which by (3.2) and (3.4) is a requirement on $z_{n-1}$ and $z_{n-2}$. After eliminating $y_{n-3}$ by (3.4) we turn (3.7) to

$$
z_{n-2}>\frac{p_{n-2}\left(y_{n-2}-y_{n}\right)+p_{n-1}\left(y_{n-1}-y_{n}\right)}{p_{n-1}\left(y_{n-2}-y_{n-1}\right)} .
$$

This is satisfied if, according to (3.5),

$$
\begin{aligned}
& \frac{p_{n-1}}{2} \frac{p_{n-2}\left(y_{n-2}-y_{n}\right)+p_{n-1}\left(y_{n-1}-y_{n}\right)}{p_{n-1}\left(y_{n-2}-y_{n-1}\right)} \\
< & \log \left(1+\frac{p_{n-2}\left(y_{n-2}-y_{n}\right)+p_{n-1}\left(y_{n-1}-y_{n}\right)}{p_{n-1}\left(y_{n-2}-y_{n-1}\right)}\right) .
\end{aligned}
$$

This simplifies to

$$
\begin{aligned}
& \log \left(1+\frac{p_{n-1}\left(y_{n}-y_{n-1}\right)+p_{n-2}\left(y_{n}-y_{n-2}\right)}{2\left(y_{n-2}-y_{n}\right)}\right) \\
< & \frac{p_{n-1}\left(y_{n}-y_{n-1}\right)+p_{n-2}\left(y_{n}-y_{n-2}\right)}{2\left(y_{n-2}-y_{n}\right)},
\end{aligned}
$$


and is true because by (3.6)

$$
\frac{p_{n-1}\left(y_{n}-y_{n-1}\right)+p_{n-2}\left(y_{n}-y_{n-2}\right)}{2\left(y_{n-2}-y_{n}\right)} \neq 0 .
$$

Continue this process until we find all positive, unique

$$
z_{i}=\frac{y_{i-1}-y_{i+1}}{y_{i+1}-y_{i}}
$$

for $i=1,2, \ldots n-1$ (Recall $y_{n}=\frac{1+m}{2}$ to define $\left.z_{n-1}\right)$. And in the mean time (3.6) and (3.7) extend to

$$
p_{0} y_{n}>p_{1}\left(y_{1}-y_{n}\right)>p_{2}\left(y_{n}-y_{2}\right)>\ldots>(-1)^{n-1} p_{n-1}\left(y_{n}-y_{n-1}\right)>0,
$$

proving the inequalities of this proposition.

Finally we write (3.8) as a linear system

$$
\left[\begin{array}{crrrr}
z_{1} & -1-z_{1} & & & \\
1 & z_{2} & -1-z_{2} & & \\
& & \cdots & & \\
& & 1 & z_{n-2} & -1-z_{n-2} \\
& & & 1 & z_{n-1}
\end{array}\right]\left[\begin{array}{c}
y_{1} \\
y_{2} \\
\cdots \\
y_{n-2} \\
y_{n-1}
\end{array}\right]=\left[\begin{array}{c}
0 \\
0 \\
\cdots \\
0 \\
\left(1+z_{n-1}\right) y_{n}
\end{array}\right]
$$

to determine the $y_{i}$ 's from the $z_{i}$ 's.

To see that the system is non-singular, we consider the homogeneous system $M \vec{h}=\overrightarrow{0}$, where $M$ is the matrix in $(3.10)$ and $\vec{h}=\left(h_{1}, h_{2}, \ldots, h_{n-1}\right)$. If $h_{1} \neq 0$, then $h_{2}$ is not zero and has the same sign. The second through the $(n-2)$ th equations imply that all the $h_{i}$ 's are nonzero and have the same sign. But then the last equation can not be satisfied. If $h_{1}=0$, then by the first $n-1$ equations, $\vec{h}=\overrightarrow{0}$. Hence the system (3.10) is non-singular.

Therefore we determine $y_{1}, y_{2}, \ldots, y_{n-1}$ uniquely from (3.10). To see that this $\left(y_{1}, y_{2}, \ldots, y_{n-1}\right)$ is in $A_{n}^{-}$, it suffices to prove that all the $y_{i}$ 's are positive, because of (3.9). Again if $y_{1}$ is $\leq 0$, then all the $y_{i}$ 's are $\leq 0$ from the first $n-2$ equations of (3.10), and the last equation can not be satisfied. So $y_{1}>0$, and the first $n-2$ equations imply that all the $y_{i}$ 's are positive.

Proposition 3.2 Any critical point of $N$ in each $A_{n}^{ \pm}$must have a positive definite Hessian. Hence it is a non-degenerate local minimum.

Proof. The second derivatives of $N$ in $A_{n}^{-}$are

$$
\begin{aligned}
\frac{\partial^{2} N}{\partial y_{1}^{2}} & =\log \frac{-y_{1}}{y_{2}-y_{1}}+\frac{y_{1}-y_{n}}{y_{1}}-\frac{y_{1}-y_{n}}{y_{1}-y_{2}}, \\
\frac{\partial^{2} N}{\partial y_{i}^{2}} & =\log \frac{y_{i-1}-y_{i}}{y_{i+1}-y_{i}}+\frac{y_{i}-y_{n}}{y_{i}-y_{i-1}}-\frac{y_{i}-y_{n}}{y_{i}-y_{i+1}}, \\
\frac{\partial^{2} N}{\partial y_{n-1}^{2}} & =\log \frac{y_{n-2}-y_{n-1}}{y_{n}-y_{n-1}}+\frac{y_{n-1}-y_{n}}{y_{n-1}-y_{n-2}}-1, \\
\frac{\partial^{2} N}{\partial y_{i} \partial y_{i+1}} & =\frac{p_{i}\left(y_{i}-y_{n}\right)+p_{i+1}\left(y_{i+1}-y_{n}\right)}{2\left(y_{i}-y_{i+1}\right)} .
\end{aligned}
$$


Note that at a critical point

$$
\begin{aligned}
\frac{\partial^{2} N}{\partial y_{i}^{2}}= & \frac{p_{i+1}\left(y_{i+1}-y_{i-1}\right)}{2\left(y_{i}-y_{n}\right)}+\frac{y_{i}-y_{n}}{y_{i}-y_{i-1}}-\frac{y_{i}-y_{n}}{y_{i}-y_{i+1}} \\
= & \frac{-p_{i}\left(y_{i}-y_{n}\right)+p_{i-1}\left(y_{n}-y_{i-1}\right)}{2\left(y_{i}-y_{i-1}\right)} \cdot \frac{y_{n}-y_{i-1}}{y_{i}-y_{n}} \\
& +\frac{p_{i}\left(y_{i}-y_{n}\right)-p_{i+1}\left(y_{n}-y_{i+1}\right)}{2\left(y_{i}-y_{i+1}\right)} \cdot \frac{y_{n}-y_{i+1}}{y_{i}-y_{n}}
\end{aligned}
$$

Take a vector $\vec{h}=\left(h_{1}, \ldots, h_{n-1}\right)$ and consider the quadratic form

$$
\begin{aligned}
\sum_{i, j=1}^{n-1} & \frac{\partial^{2} N}{\partial y_{i} \partial y_{j}} h_{i} h_{j} \\
= & \sum_{j=1}^{n-1} \frac{\partial^{2} N}{\partial y_{j}^{2}} h_{j}^{2}+2 \sum_{j=2}^{n-1} \frac{\partial^{2} N}{\partial y_{j} y_{j-1}} h_{j} h_{j-1} \\
= & \sum_{i=2}^{n-1} \frac{-p_{i}\left(y_{i}-y_{n}\right)+p_{i-1}\left(y_{n}-y_{i-1}\right)}{2\left(y_{i}-y_{i-1}\right)}\left(\sqrt{\frac{y_{n}-y_{i-1}}{y_{i}-y_{n}}} h_{i-1}-\sqrt{\frac{y_{i}-y_{n}}{y_{n}-y_{i-1}}} h_{i}\right)^{2} \\
& +\frac{-p_{1}\left(y_{1}-y_{n}\right)+p_{0} y_{n}}{2 y_{1}} \cdot \frac{y_{n}}{y_{1}-y_{n}} h_{1}^{2}
\end{aligned}
$$

by (3.11). This quantity is positive when $\vec{h} \neq \overrightarrow{0}$ by the inequalities in Proposition 3.1. Thus $N$ is positive definite at the critical point.

Proposition 3.3 The lone members in $A_{1}^{ \pm}$are local minima of $I_{0}$ for any positive $\gamma$. In $A_{n}^{ \pm}, n \geq 2, I_{0}$ has a strict local minimum if $\gamma$ is large.

Proof. The lone elements in $A_{1}^{ \pm}$are considered minima for any $\gamma>0$. In $A_{n}^{ \pm}(n \geq 2)$, the proposition follows from Propositions 3.1 and 3.2 , since nondegenerate local minima of $N$ persist under perturbation to $L+N$ when $c$ is small, i.e. $\gamma$ is large.

The largeness condition in this proposition can not be removed. For instance in $A_{2}^{-}$with $m=0$,

$$
\frac{1}{\gamma \pi} \frac{\partial I_{0}}{\partial y_{1}}=\frac{c}{2}\left(\frac{1}{\sqrt{y_{1}}}+\frac{1}{\sqrt{y_{1}-0.5}}\right)-\frac{1}{4}+\left(y_{1}-0.5\right) \log \frac{-y_{1}}{0.5-y_{1}}
$$

is positive in $(0.5,1)$ if $c$ is large, i.e. $\gamma$ is small. Then there is no local minimum. In general the local part $L$ of $I_{0}$ is concave in $A_{n}^{ \pm}$, because

$$
\sum_{i, j=1}^{n-1} \frac{\partial^{2} L}{\partial y_{i} \partial y_{j}} h_{i} h_{j}=-\frac{c}{4}\left[\sum_{i=2}^{n-1}\left|y_{i}-y_{i-1}\right|^{-\frac{3}{2}}\left(h_{i}-h_{i-1}\right)^{2}+y_{1}^{-\frac{3}{2}} h_{1}^{2}+\left|y_{n-1}-y_{n}\right|^{-\frac{3}{2}} h_{n-1}^{2}\right]
$$

is negative if $\left(h_{1}, h_{2}, \ldots, h_{n-1}\right) \neq \overrightarrow{0}$. Then $I_{0}$ is concave if $\gamma$ is small. Since $A_{n}^{ \pm}$ do not include their boundaries (The boundaries of $A_{n}^{ \pm}$consist of $A_{m}^{ \pm}, m<n$ ), there is no local minimum in $A_{n}^{ \pm}$. 

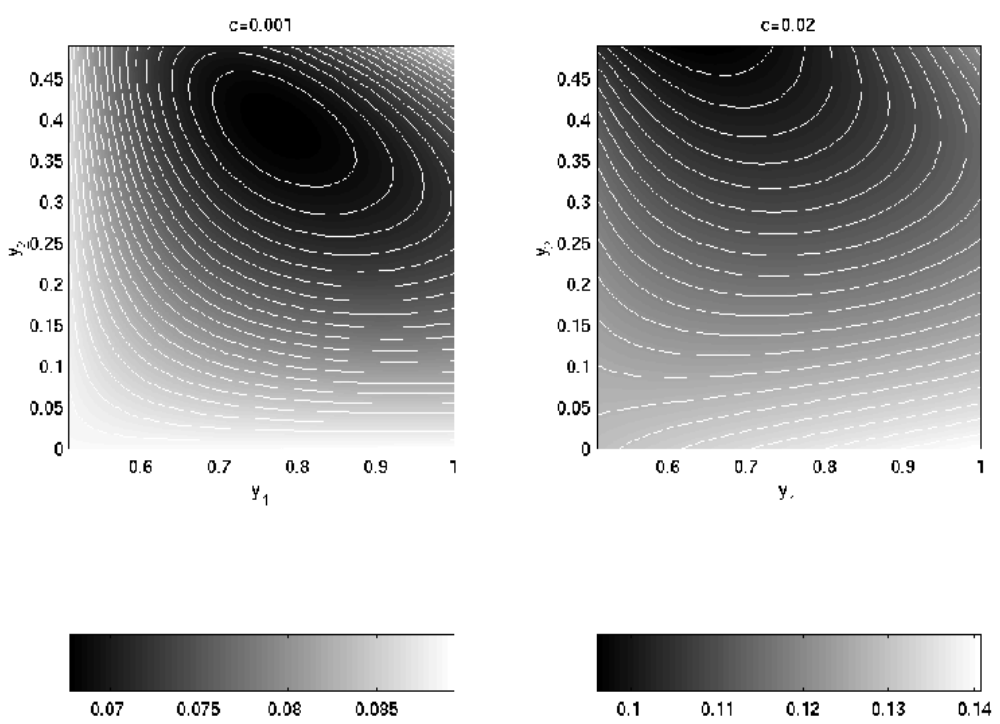

Figure 1: Contour maps of $\frac{1}{\gamma \pi} I_{0}$ in $A_{3}^{-1}$.

In $A_{3}^{-}$, with $m=0$, the two plots in Figure 1 give the contour maps of $\frac{1}{\gamma \pi} I_{0}$ when $\gamma$ is large, where there is a local minimum, and when $\gamma$ is small, where there is no local minimum.

Although $N$ has only one critical point in each $A_{n}^{ \pm}, I_{0}$ may have more. In $A_{2}^{-}$, when $\gamma$ is large, $I_{0}$ has a local maximum before the local minimum, mentioned in Proposition 3.3. The first plot of Figure 2 shows the graph of $\frac{1}{\gamma \pi} I_{0}$ vs. $y_{1}$ with a large $\gamma$, and the second plot enlarges a portion of this picture so we can see a local maximum followed by a local minimum. On the contrary in the one-dimensional case $\Omega=(0,1)$, the $\Gamma$ limit has a unique critical point, which is a local minimum in each set of functions with $n$ jumps (See [19]).

We remark at this point that if we study the Cahn-Hilliard problem in the current setting, there is no local minimum of the corresponding $\Gamma$-limit in any $A_{n}^{ \pm}, n \geq 2$, simply because of the absence of $N$ and the concavity of $L$.

Proof of Theorem 1.1. This follows immediately from Propositions 2.2, 2.3 and 3.3 . 

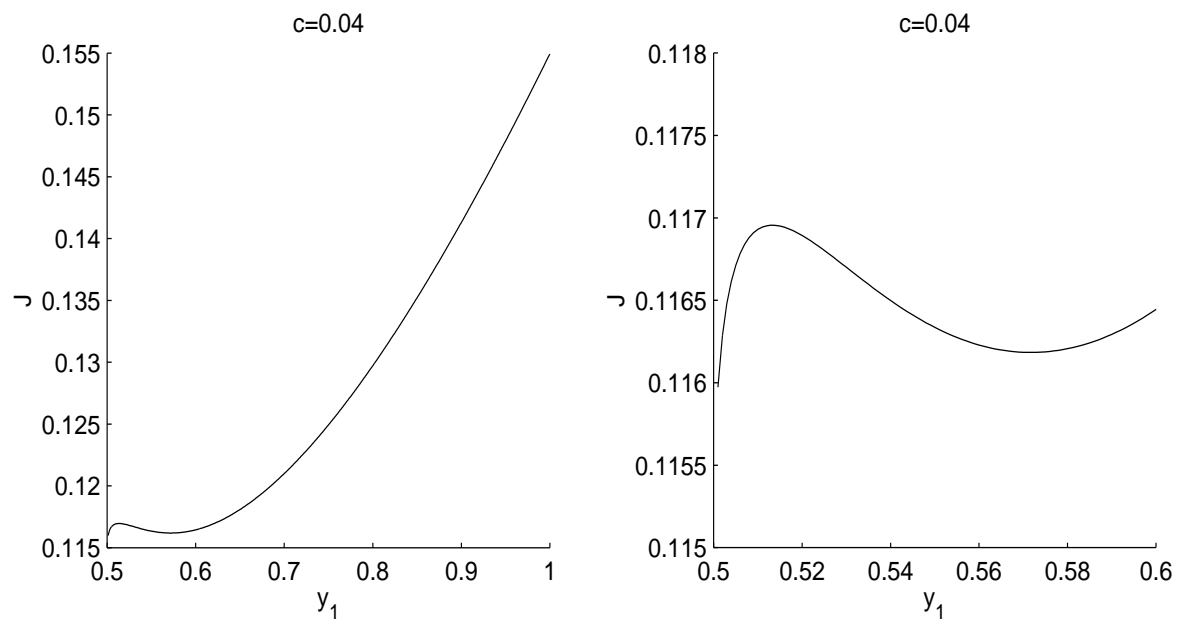

Figure 2: Graph of $\frac{1}{\gamma \pi} I_{0}$ in $A_{2}^{-1}$.

\section{Layer disappearance}

We turn our attention to the dynamics of the copolymer problem.

A simple dynamic law of $F,(1.1)$, is the forth order partial differential equation

$$
\epsilon u_{t}=\Delta\left(-\epsilon^{2} \Delta u+f(u)\right)-\sigma(u-m)
$$

under the boundary conditions

$$
\left.\frac{\partial u}{\partial \nu}\right|_{\partial \Omega}=\left.\frac{\partial \Delta u}{\partial \nu}\right|_{\partial \Omega}=0 .
$$

This is a diffusive model where diffusion is driven by the the chemical force from the free energy $F$. It ignores the kinetics of motion.

In the asymptotic regime $\sigma \sim \epsilon$ where we have the $\Gamma$-limit (2.1), there is a corresponding dynamic singular limit, identified by Nishiura and Ohnishi in [15]. It is a free boundary problem (FBP) which generalizes the one associated with the Cahn-Hilliard problem (see e.g. Pego [18]). Suppose that $S$ is the set of interfaces separating $\Omega$ into $\Omega^{-}$where $u=-1$ and $\Omega^{+}$where $u=1$. Then it evolves under the following law:

$$
\begin{aligned}
\Delta h & =0 \quad \text { in } \Omega^{ \pm} \\
\frac{\partial h}{\partial \nu} & =0 \text { on } \partial \Omega \\
V & =-\frac{1}{2}\left[\frac{\partial h}{\partial \nu}\right] \quad \text { on } S \\
\frac{c K}{4} & =h-(-\Delta)^{-1}(u-m) \text { on } S,
\end{aligned}
$$


where $V$ is the normal velocity, $K$ the mean curvature of $S$, and $c$ the same constant (2.7). The normal direction $\nu$ of $S$ in (4.5) is towards $\Omega^{+}$. [...] there denotes the jump of the directional derivative. $V$ is positive if motion is in the same direction. The curvature is counted positive if the center of curvature lies on the $\Omega^{+}$side.

The convergence of (4.1-4.2) to (4.3-4.6) in the class of radial functions was shown in Henry [9].

Applied to the the radially symmetric case $I_{0}$ in $A_{n}^{-}$, the FBP takes a simpler form: when the radii of the interfaces are denoted by $r_{1}, r_{2}, \ldots, r_{n}$, they satisfy a system of differential equations

$$
\begin{aligned}
\frac{d r_{i}}{d t}= & \frac{-\frac{c}{4}\left(\frac{1}{r_{i-1}}+\frac{1}{r_{i}}\right)-(-1)^{i}\left[v\left(r_{i-1}\right)-v\left(r_{i}\right)\right]}{2 r_{i} \log \frac{r_{i}}{r_{i-1}}} \\
& +\frac{-\frac{c}{4}\left(\frac{1}{r_{i+1}}+\frac{1}{r_{i}}\right)+(-1)^{i}\left[v\left(r_{i}\right)-v\left(r_{i+1}\right)\right]}{2 r_{i} \log \frac{r_{i}}{r_{i+1}}}
\end{aligned}
$$

where $v=(-\Delta)^{-1}(u-m)$. When $i=1$ the first term on the right side of (4.7) is absent, and when $i=n$ the second term is absent. With respect to $y$-coordinates, the system (4.7) becomes

$$
\begin{aligned}
\frac{d y_{i}}{d t} & =-\frac{\frac{(-1)^{i-1} c}{2 \sqrt{\left|y_{i-1}-y_{i}\right|}}+\frac{(-1)^{i+1} c}{2 \sqrt{\left|y_{i+1}-y_{i}\right|}}+\frac{p_{i+1}\left(y_{i-1}-y_{i+1}\right)}{2}+\left(y_{i}-y_{n}\right) \log \frac{y_{i-1}-y_{i}}{y_{i+1}-y_{i}}}{\log \frac{y_{i-1}-y_{i}}{y_{i+1}-y_{i}}} \\
& =-\frac{1}{\gamma \pi \log \frac{y_{i-1}-y_{i}}{y_{i+1}-y_{i}}} \cdot \frac{\partial I_{0}}{\partial y_{i}},
\end{aligned}
$$

for $i=1,2, \ldots, n-1$.

Along a solution $y_{i}(t)$ of $(4.8)$

$$
\frac{d I_{0}}{d t}=-\sum_{i=1}^{n-1} \frac{1}{\gamma \pi \log \frac{y_{i-1}-y_{i}}{y_{i+1}-y_{i}}} \cdot\left(\frac{\partial I_{0}}{\partial y_{i}}\right)^{2} .
$$

The log terms are all positive by (2.9), so $I_{0}$ is a Lyapunov functional of (4.8).

Several issues including interface disappearance may be addressed by the properties of $I_{0}$.

Convergence to the equilibria. Take an initial configuration $u \in A_{2}^{-}$. $u$ has two circular interfaces $r_{1}$ and $r_{2}$. If $u$ is close to the local minimum of $I_{0}$, depicted in Figure 2, then under the FBP $u$ converges to this local minimum. Same convergence results hold for the local minima of $I_{0}$ in other $A_{n}^{ \pm}$.

Disappearance at the origin. However if we take the initial $u \in A_{2}^{-}$in such a way that $r_{1}$ of $u$ is slightly smaller than the $r_{1}$-coordinate of the local maximum in Figure 2, then $r_{1}$ moves towards $\frac{1+m}{2}$ and $r_{2}$ towards 0 . This way the inner 
interface $r_{2}$ shrinks to 0 and disappears. In other words $u$ in $A_{2}^{-}$moves to a boundary point of $A_{2}^{-}$, which is the lone element of $A_{1}^{-}$.

Here we make a note that according to Fife and Hilhorst [8] interfaces do not disappear or emerge in the FBP when $\Omega=(0,1)$, due to the absence of the curvature term in (4.6).

The local maximum of $I_{0}$ in $A_{2}^{-}$depicted in Figure 2 is a saddle point of $I_{0}$ in $A$. We speculate that it also corresponds to a saddle point of $I_{\epsilon}$, with a one-dimensional unstable manifold. However the $\Gamma$-convergence theory is not applicable in this situation.

More can be said from Figure 2. We note that letting $r_{2} \searrow 0$ is the only way for $u$ to leave $A_{2}^{-}$. Since the energy $I_{0}$ goes up as $r_{1} \nearrow 1$, we can not lose the outer interface $r_{1}$ by having $r_{1} \nearrow 1$. So $u$ can not move to the second boundary point of $A_{2}^{-}$, which is the lone element in $A_{1}^{+}$.

This type of interface disappearance may occur in other $A_{n}^{ \pm}$as well. A initial $u$ starting in $A_{n}^{-}$(or $A_{n}^{+}$respectively) may move to a boundary point, which is a member in some $A_{m}^{-}$(or $A_{m}^{+}$respectively) with $m<n$.

Interface collapsing. Another interesting question is whether the FBP may lose interfaces by collapsing some of them into each other. This actually can happen. For example we take $A_{3}^{-}$and consider the case that $r_{1}, r_{2}$ and $r_{3}$ are all close to each other. In terms of $y_{1}$ and $y_{2}$ this means $y_{1} \searrow \frac{1+m}{2}$ and $y_{2} \searrow 0$. Compute

$$
\frac{1}{\gamma \pi} \nabla I_{0}\left(y_{1}=y_{3}, y_{2}=0\right)=\left(\frac{c}{\sqrt{y_{3}}},-\frac{c}{\sqrt{y_{3}}}\right) .
$$

This gradient vector and the ones nearby all point to the southwest direction. It implies that any initial $u$ in this neighbourhood will move to the $y_{2}$ axis, causing the $r_{1}$ circle to move to $\sqrt{\frac{1+m}{2}}$ and the $r_{2}$ and $r_{3}$ circles to collapse to each other and disappear.

Nucleation. There is also the issue of nucleation. Here nucleation is the opposite of interface disappearance. Can the FBP create interfaces? In general the answer is no. This is because creating interfaces away from the origin causes $I_{0}$ to jump up, and creating interfaces near the origin, as opposite to losing interfaces, which was discussed earlier, also increases $I_{0}$. This was essentially illustrated in the proof of Proposition 2.3.

\section{Discussion}

Our study of concentrically layered equilibria of $I_{\epsilon}$ revealed how curved interfaces behave in the di-block copolymer problem.

We first considered the static problem by seeking local minima of the free energy $I_{\epsilon}$ in the class of radial functions. Analyzing the singular limit $I_{0}$ of $I_{\epsilon}$ we constructed equilibria of $I_{\epsilon}$ with one circular interface when $\epsilon \sim \sigma$ are both small. 
To find equilibria of $I_{\epsilon}$ with multiple circular interfaces we needed to impose an extra condition that $\gamma=\frac{\sigma}{\epsilon}$ is large. This contrasts with the parallel interface situation, where equilibria with multiple interfaces always exist regardless of the value of $\gamma$.

A related question is to fix a large $\gamma$ and ask how many multiply layered equilibria exist and how many layers there are. To answer this question we have to study the graph of $I_{0}$ in each $A_{n}^{ \pm}$, and find the $A_{n}^{ \pm}$'s where $I_{0}$ has local minima. It is a quite tedious task due to the complex formula (2.8) or (2.10). We believe that there should be two positive integers $N_{\gamma}^{ \pm} \geq 1$, such that if and only if $n \leq N_{\gamma}^{-}\left(n \leq N_{\gamma}^{+}\right.$respectively $)$, there is a local minimum in $A_{n}^{-}\left(A_{n}^{+}\right.$ respectively).

When such a layered equilibrium exists, the location of the interfaces is determined by the $r$-coordinates of the local minimum of $I_{0}$ in $A_{n}^{ \pm}$. Unlike the case of parallel interfaces (See [19]), the circular interfaces do not appear periodically. This suggests the role that the different curvatures $\left(\frac{1}{r_{i}}\right.$ when the radii are $r_{i}$ ) of the interfaces play in this problem.

To better understand the curvature factor we turned to a dynamic law associated with the functional $I_{\epsilon}$. It has a free boundary problem as a singular limit and the functional $I_{0}$ serves as a Lyapunov functional of this limit. In this dynamic problem, the curvatures of the interfaces enter the equation. In addition to the observation of convergence to the layered equilibria, we found initial elements in $A$ that lead to interface disappearance at the origin, or interface collapsing to each other. These two phenomena contrast nicely with the case of parallel interfaces $(\Omega=(0,1))$, where neither of them exists.

\section{References}

[1] M. Bahiana And Y. Oono, Cell dynamical system approach to block copolymers, Phys. Rev. A, 41 (1990), 6763-6771.

[2] F.S. BAtes And G.H. Fredrickson, Block copolymers - designer soft materials, Physics Today, 52-2 (Feb, 1999), 32-38.

[3] J.W. Cahn and J.E. Hilliard, Free energy of a nonuniform system, I. Interfacial free energy, J. Chem. Phys. 28 (1958), 258-267.

[4] R. Choksi, Scaling laws in microphase separation of diblock copolymers, J. Nonlinear Sci., 11 (2001), 223-236.

[5] G. Dal Maso, An Introduction to $\Gamma$-convergence, Progress in Nonlinear Diff. Eqn. and Appl., Birkhäuser, Boston 1993.

[6] E. De Giorgi, Sulla convergenza di alcune successioni di integrali del tipo della'area, Rendiconti di Matematica, 8 (1975), 277-294.

[7] L. Evans And R. Gariepy, Measure Theory and Fine Properties of Functions, CRC Press, Boca Raton New York London Tokyo, 1992. 
[8] P.C. Fife And D. Hilhorst, The Nishiura-Ohnishi free boundary problem in the 1D case, SIAM J. Math. Anal., 33 (2001), 589-606.

[9] M. Henry, Singular limit of a fourth order problem arising in the microphase separation of diblock copolymers, Adv. Differential Equations, 6 (2001), 1049-1114.

[10] R. Kohn and P. Sternberg, Local minimisers and singular perturbations, Proc. Royal Soc. Edin., 111A (1989), 69-84.

[11] L. LEIBLER, Theory of microphase separation in block copolymers, Macromolecules 13 (1980), 1602-1617.

[12] L. ModicA, The gradient theory of phase transitions and the minimal interface criterion, Arch. Rat. Mech. Anal., 98 (1987), 357-383.

[13] L. Modica And S. Mortola, Un esempio di $\Gamma^{-}$-convergenza, Boll. Un. Mat. Ital. B (5), 14 (1977), 285-299.

[14] S. MüLLER, Singular perturbations as a selection criterion for periodic minimizing sequences, Calc. Var. Partial Differential Equations 1-2 (1993), 169-204.

[15] Y. Nishiura and I. Ohnishi, Some mathematical aspects of the microphase separation in diblock copolymers, Physica D 84 (1995), 31-39.

[16] I. Ohnishi, Y. Nishiura, M. Imai and Y. Matsushita, Analytical solutions describing the phase separation driven by a free energy functional containing a long-range interaction term, Chaos 9-2 (1999), 329-341.

[17] T. OHTA AND K. KaWASAKI, Equilibrium morphology of block copolymer melts, Macromolecules, 19 (1986), 2621-2632.

[18] R.L. Pego, Front migration in the nonlinear Cahn-Hilliard equation, Proc. Roy. Soc. London Ser. A 422 (1989), 261-278.

[19] X. Ren And J. Wei, On the multiplicity of solutions of two nonlocal variational problems, SIAM J. Math. Anal. 31 (2000), 909-924.

[20] X. Ren and J. Wei, On energy minimizers of the di-block copolymer problem, preprint.

[21] J. Wei And M. Winter, On the stationary Cahn-Hilliard equation: Bubble solutions, SIAM J. Math. Anal. 29 (1998), 1492-1518. 